This item is the archived peer-reviewed author-version of:

Myasthenia gravis appearing after thymectomy heralding recurrent thymoma

\title{
Reference:
}

Beckers Paul, Mercelis Rudolf, Heyman Stijn, Verheuen Lies, Lauw ers Patrick, Hendriks Jeroen, van Schil Paul.- Myasthenia gravis appearing after thymectomy heralding recurrent thymoma

Acta chirurgica Belgica - ISSN 0001-5458 - (2018), p. 1-3

Full text (Publisher's DOI): https://doi.org/10.1080/00015458.2018.1438556 


\section{Myasthenia Gravis Appearing After Thymectomy Heralding Recurrent Thymoma.}

Paul AJ Beckers ${ }^{1}$, Rudy Mercelis ${ }^{2}$, Stijn Heyman ${ }^{3}$, Lies Verheuen ${ }^{4}$, Patrick Lauwers $^{1}$, Jeroen M Hendriks ${ }^{1}$, Paul E Van Schil ${ }^{1}$

${ }^{1}$ Department of Thoracic and Vascular Surgery, Antwerp University Hospital, Edegem, Belgium

${ }^{2}$ Department of Neurology, Antwerp University Hospital, Edegem, Belgium

${ }^{3}$ Department of General Surgery, Antwerp Hospital Network (ZNA), Antwerp, Belgium

${ }^{4}$ Department of Pathology, Antwerp University Hospital, Edegem, Belgium

Corresponding author: Paul Beckers, Department of Thoracic and Vascular Surgery, Antwerp University Hospital, Wilrijkstraat 10, 2650 Edegem, BELGIUM; Email: paul.beckers2@uantwerpen.be

Funding: There was no financial support for this article

Disclosure: The authors report no conflicts of interest 


\section{Myasthenia Gravis Appearing After Thymectomy Heralding Recurrent Thymoma.}

Introduction 30 to $50 \%$ of thymoma patients develop myasthenia gravis. In 1,5$28 \%$ of cases myasthenia gravis appears many years after removal of a thymoma.

Patients and methods We present a case report of a 72-year-old female who presented with myasthenia gravis four months after total thymectomy.

Results A 72-year-old female patient presents with myasthenia gravis four months after total thymectomy. Imaging revealed a PET-positive nodule anterior to the superior vena cava. By median sternotomy the nodule was removed at our hospital. Pathology confirmed a recurrent B2/B3 thymoma with R0 resection. No adjuvant therapy was given. Large population studies show the appearance of new-onset myasthenia gravis associated with recurrent thymoma in 3\% of cases.

Conclusion New-onset myasthenia gravis postthymectomy heralds recurrent disease in $3 \%$ of cases. Thorough screening is needed in such patients.

Keywords: Myasthenia gravis; thymoma; thymectomy; recurrent disease; sternotomy

\section{Introduction}

Thymomas are epithelial tumors of the thymus and account for approximately $50 \%$ of the masses in the anterior mediastinum (1). Most series report that $30 \%$ to $50 \%$ of thymoma patients have myasthenia gravis (MG), while $10-15 \%$ of $\mathrm{MG}$ patients present with a thymoma $(2,3)$. In $1.5-28 \%$ of cases MG appears many years after removal of a thymoma $(4,5)$.

\section{Case report}

A 72-year-old female presented at a peripheral hospital with progressive fatigue and shortness of breath during minimal exercise. Clinical examination showed paleness and icteric sclerae. A Coombs positive (anti-IgG positive, anti-C3d negative) auto-immune 
hemolytic anemia was discovered. An anterior mediastinal tumor adherent to the right atrium was found on chest computed tomography (CT). This nodule was slightly fluorodeoxyglucose (FDG)-positive on positron emission tomographic (PET) scanning. Thoracoscopic removal of the mass was performed which showed a thymoma type B2, WHO classification. Pathological examination showed macroscopic invasion of the pericardium (stage III, Masoaka-Koga [M-K] classification (6)). Because of suspicious pericardial margins a revision by right thoracotomy with pericardectomy was performed one month after the original operation. Pathology showed no residual tumor. Four months after the revision she complained of problems with swallowing and fatigue during exercise. After eight months of progressive complaints the diagnosis of MG was made by detecting acetylcholine receptor-antibodies (18.01 nmol/L). CT showed a nodule anterior to the superior vena cava (fig. 1) which was PET-positive. She was then referred to our hospital. By median sternotomy the nodule was removed including the surrounding mediastinal fat. Pathology confirmed a recurrent B2/B3 thymoma (WHO classification, fig. 2) with R0 resection. Adjuvant radiotherapy was refused by the patient, MG was treated successfully with Methylprednisolon and Pyridostigmin.

\section{Discussion}

Thymoma is the most frequent tumor in the anterior mediastinum. MG is often associated with thymoma at initial presentation of the tumor but may be diagnosed some variable time after removal of the thymus (so-called postthymectomy myasthenia gravis [PTMG]) (3). Kondo et al (4) reported PTMG in 0.97\% (8/827) thymoma patients. Namba et al (7) and Ito et al (8) reported 3\% and 3.3\% PTMG patients respectively. Bae et al (9) reported 2.8\% (11/391) PTMG patients while half of these patients had recurrent tumors (PTMG associated to recurrent thymoma (PTMG-ART)). 
Other reports of PTMG-ART show evidence of metastatic disease. A 49-year-old patient presented with PTMG one year after total resection of thymic tissue. She died 21 months later of myasthenic crisis. Autopsy revealed a metastatic thymoma in the left lung (10). Another case consisted of a 69-year old patient with a myasthenic crisis 22 years after resection of the primary tumor. Examination showed a solitary liver metastasis (11).

Due to its malignant potential, complete resection is the gold standard for treatment of thymoma (12). A recent review showed that repeat thymectomy should be attempted for patients with refractory MG after a previous thymectomy. Though complete remissions are rare, $60-70 \%$ cases report clinical improvement (13).

Conclusion Our patient presented with an incidental diagnosis of an asymptomatic thymoma with complete resection of a stage III tumor. MG occurred within one year after surgery associated with a local recurrence of the thymoma. Approximately $3 \%$ of new-onset myasthenia gravis postthymectomy is associated with recurrent disease. Therefore thorough screening is needed when a patient presents with postthymectomy myasthenia gravis.

1. Detterbeck FC, Zeeshan A. Thymoma: current diagnosis and treatment. Chinese medical journal. 2013;126(11):2186-91.

2. Ruffini E, Venuta F. Management of thymic tumors: a European perspective. Journal of thoracic disease. 2014;6 Suppl 2:S228-37.

3. Nakajima J, Murakawa T, Fukami T, Sano A, Takamoto S, Ohtsu H. Postthymectomy myasthenia gravis: relationship with thymoma and antiacetylcholine receptor antibody. The Annals of thoracic surgery. 2008;86(3):941-5.

4. Kondo K, Monden Y. Myasthenia gravis appearing after thymectomy for thymoma. European journal of cardio-thoracic surgery : official journal of the European Association for Cardio-thoracic Surgery. 2005;28(1):22-5. 
5. Kang SY, Lee JS, Choi JC, Kang JH. Myasthenia gravis appearing after thymectomy: a case report and review of the literature. Journal of clinical neurology. 2007;3(3):158-60.

6. Detterbeck FC, Nicholson AG, Kondo K, Van Schil P, Moran C. The Masaoka-Koga stage classification for thymic malignancies: clarification and definition of terms. Journal of thoracic oncology : official publication of the International Association for the Study of Lung Cancer. 2011;6(7 Suppl 3):S1710-6.

7. Namba T, Brunner NG, Grob D. Myasthenia gravis in patients with thymoma, with particular reference to onset after thymectomy. Medicine. 1978;57(5):411-33.

8. Ito M, Fujimura S, Monden Y, Watanabe Y, Shimizu N, Hirono T, et al. [A retrospective group study on post-thymectomy myasthenia gravis]. [Zasshi] [Journal] Nihon Kyobu Geka Gakkai. 1992;40(2):189-93.

9. Abstract from the 5th International Thymic Malignancy Interest Group Annual Meeting, September 5-6, 2014, Antwerp, Belgium. Journal of thoracic oncology : official publication of the International Association for the Study of Lung Cancer. 2014;9(10 Suppl 4):S212-43.

10. Akimaru K, Shoji T, Masugi Y. A case report of post-thymectomy myasthenia gravis with residual thymoma. Nihon Ika Daigaku zasshi. 1993;60(4):241-4.

11. Denayer MA, Rao KR, Wirz D, McNally D. Hepatic metastatic thymoma and myasthenia gravis twenty-two years after the apparent cure of an invasive thymoma. A case report and review of the literature. Journal of the neurological sciences. 1986;76(1):23-30.

12. Ruffini E, Mancuso M, Oliaro A, Casadio C, Cavallo A, Cianci R, et al. Recurrence of thymoma: analysis of clinicopathologic features, treatment, and outcome. The Journal of thoracic and cardiovascular surgery. 1997;113(1):55-63.

13. Ng JK, Ng CS, Underwood MJ, Lau KK. Does repeat thymectomy improve symptoms in patients with refractory myasthenia gravis? Interactive cardiovascular and thoracic surgery. 2014;18(3):376-80. 
Figure 1. Computed tomography (CT) finding of local recidive mass of the anterior mediastinum consistent with local recurrent thymoma.

Figure 2: Macroscopic (left) and microscopic (right) view of the resected mass. Pathologic evidence of B2 (top right) and B3 (bottom right) zones. 